\title{
The Effect of Infrapatellar Fat Pad Resection in Total Knee Replacement on Patella Tendon Length and Functional Outcomes after Five Years
}

\section{Sheldon M*, Siamak S, Kieran B and Richard B \\ Orthopaedics, Royal Perth Hospital, Australia}

*Corresponding author: Sheldon Moniz (MBBS), Orthopaedics, Royal Perth Hospital, Australia Victoria Square, Perth WA 6000, Australia, Tel: 0433828015; Email: monizsheldon@gmail.com

\section{Research Article \\ Volume 3 Issue 4}

Received Date: June 12, 2019

Published Date: October 29, 2019

DOI: $10.23880 /$ jobd-16000187

\section{Abstract}

Background: It remains unclear whether infrapatellar fat pad (IPFP) excision leads to patella tendon shortening. Shortening of the tendon alters the biomechanics of the knee and can be problematic however current literature is divided regarding the effect of IPFP resection on patella length and functional outcomes following total knee replacement. Methods: Our aim was to identify any functional and radiographic differences incurred with IPFP resection. We conducted a retrospective study comparing resection and preservation of the infrapatellar fat pad between 2007-2009 using the Stryker Triathlon or Duracon implants between two independent consultant surgeons. 168 consecutive patients were recruited. Baseline demographics were similar between resection and preservation groups with a mean age of 68.5 (70 vs 67, p=0.17), BMI 31.71 (31.02 vs 32.4, p=0.84) and patella resurfacing $23.5(24 \mathrm{vs} 23$, p=0.29).

Results: Comparisons between the resection and preservation groups for the Insall-Salvati, Modified Insall-Salvati, Caton-deschamps and Blackburne-peel ratios preoperatively, at one year and five years postoperatively did not demonstrate any statistically significant differences. Knee society scores (KSS) preoperatively (45.36 vs 40.5 , p=0.31) and at five years postoperatively ( 90.49 vs $85.31, \mathrm{p}=0.2$ ) were not statistically different. Similarly anterior knee pain scores (AKPS) demonstrated no statistical significance between groups preoperatively $(50.6$ vs $42.8, p=0.3)$ and at five years post operatively (69.4 vs 62.0, p=0.88).

Conclusion: There is no statistical difference in radiological measures or functional scores (AKPS \&KSS) at one or five years post-operatively between resection or preservation of the infrapatellar fat pad following total knee arthroplasty for primary osteoarthritis.

Keywords: Infrapatellar Fat Pad; Knee Replacement; Royal Perth Hospital 


\section{Journal of Orthopedics \& Bone Disorders}

\section{Introduction}

The infrapatellar fat pad (IPFP) is an extrasynovial structure consisting of fibrous adipose tissue and is situated in the anterior compartment of the knee joint, between the joint capsule and the synovium. The IPFP may be entirely or partially excised during total knee replacement (TKR) to facilitate the surgeon's view of the lateral tibial plateau. Joint Registry data from the United Kingdom (UK) showed that $27 \%$ of surgeons excise the IPFP in its entirety, 59\% partially resect it, and $14 \%$ leave the IPFP intact [1].

It remains unclear whether IPFP excision leads to patella tendon shortening. Patellar tendon shortening is known to occur after surgery on the knee [2-8]. Shortening of the tendon alters the biomechanics of the knee and can be problematic. It is directly related to anterior knee pain and decreased range of motion after TKR [5]. It can cause patella impingement on the tibia in full flexion. Patella infera is a cause of patellofemoral pain, stiffness, and reduced functional scores after knee replacement [9].

The pathophysiology of postoperative patellar tendon shortening is believed to be multifactorial [10]. It may be due to intrinsic collagen contraction, scarring and contraction of peripatellar soft tissues, ischemic contracture of the tendon, or quadriceps weakness from prolonged knee immobilization or pain inhibition $[10,11]$. It has been shown that complete IPFP excision can devascularise the patella by interrupting the infrapatellar anastomosis $[12,13]$. This same anastomosis supplies the patella tendon.

The clinical outcome of TKR is dependent on numerous factors including patient selection, prosthesis design, surgical technique and rehabilitation programs. The surgical technique and subsequent tissue handling probably influence prospective rehabilitation and symptoms. Resection of IPFP during TKR may have short and long-term effects on clinical outcomes [14-19].

Recent systematic review articles have highlighted the paucity of data on the effect of IPFP resection on patient satisfaction scores $[19,20]$. Only one retrospective study was identified, which looked at 8231 TKRs in the UK and found no significant difference in patient satisfaction across three groups of patients who had either complete resection, partial resection or preserved IPFP [21].
To our knowledge, there are no evidence-based consensus guidelines on whether to respect the IPFP during TKR.

\section{Aim and Methods}

Our aim was to compare patellar tendon length and functional outcome scores after knee arthroplasty for osteoarthritis, between patients in whom the infrapatellar fat pad was completely excised and those in whom it was preserved.

We conducted a retrospective study looking at resection versus preservation of the infrapatellar fat pad in primary total knee replacements. Consecutive cases were identified via the Joint Replacement Arthroplasty Clinic (JRAC) Database at Royal Perth Hospital between 2007-2009 using the Stryker Triathlon or Duracon implants between two independent consultant surgeons.

Sample size calculations based on the study by Meneghini, et al. [14] estimated 54 patients in each group to have an $80 \%$ chance of detecting a $5 \%$ difference in change of length of the patellar tendon between the groups at a $5 \%$ level of significance. Patients with primary osteoarthritis aged 18 years or older were included in this study. The exclusion criteria were inadequate radiographs, previous knee surgery (High Tibial Osteotomy, ACL reconstruction, etc.), inflammatory arthropathy, postoperative sepsis, intraoperative tibial plateau fractures, bilateral total knee replacement and intraoperative lateral release.

168 consecutive records were reviewed 77 resection vs 91 preserved) with true lateral xray taken at 30 degrees of flexion. Imaging was taken preoperatively, $1 \mathrm{yr}$ post operatively and 5yrs post operatively and reviewed by three independent clinicians blinded with the mean patella tendon ratio recorded. Functional measures were recorded by independent musculoskeletal physiotherapists through the JRAC program.

\section{Results}

168 consecutive patients were retrospectively recruited into this study. Baseline demographics were similar between resection and preservation groups with a mean age of 68.5 ( 70 vs 67, $\mathrm{p}=0.17$ ), BMI 31.71 (31.02 vs $32.4, \mathrm{p}=0.84$ ) and patella resurfacing 23.5 (24 vs 23, $\mathrm{p}=0.29)$. 


\begin{tabular}{|c|c|c|c|}
\hline & Resection & Preservation & P-Value \\
\hline Age $\overline{\mathbf{x}}$ & 70 & 67 & 0.17 \\
\hline BMI $\overline{\mathbf{x}}$ & 31.02 & 32.4 & 0.84 \\
\hline $\begin{array}{c}\text { Patella } \\
\text { Resurfaced }\end{array}$ & 24 & 23 & 0.29 \\
\hline
\end{tabular}

Table 1: Demographic Data (total patient cohort).

A comparison was made between the resection and preservation groups for the Insall-Salvati ratio preoperatively ( 0.98 vs $0.99, \mathrm{p}=0.77)$, at one year postoperatively ( 0.97 vs $0.97, \mathrm{p}=0.87$ ) and five years postoperatively $(0.91$ vs $0.92, \quad \mathrm{p}=0.75)$.No statistical difference was found at any time period (Figure 1).

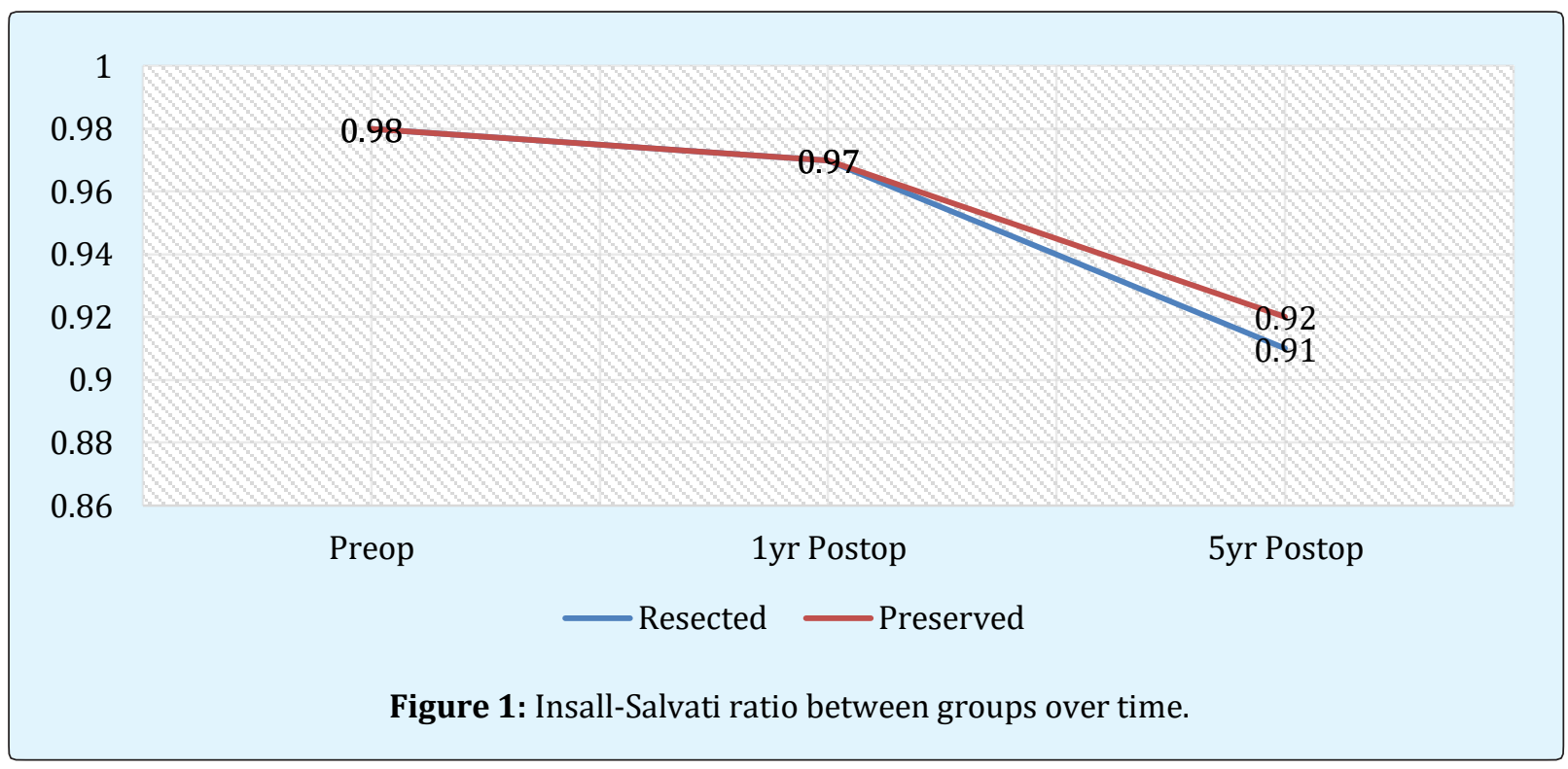

Similarly no difference was observed for the Modified Insall-Salvati ratio preoperatively ( 1.42 vs $1.46 \mathrm{p}=0.17$ ),

at one year postoperatively ( 1.36 vs $1.40, \mathrm{p}=0.15)$ and five years postoperatively ( 1.30 vs $1.34, \mathrm{p}=0.23$ ) (Figure 2 ).

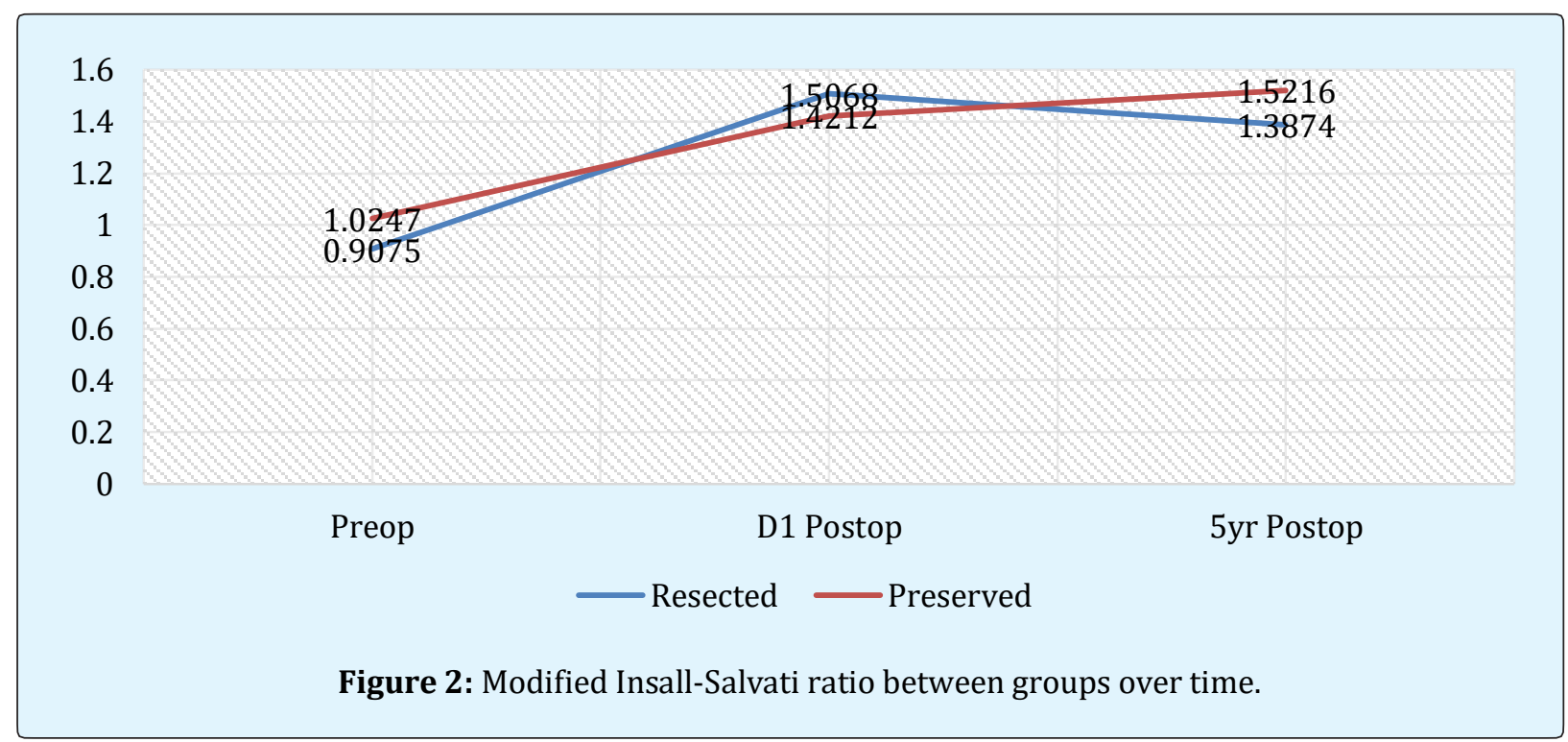


Whilst statistically significant differences were detected with the Blackburne-peel ratio at one year post operatively (0.82 vs $0.77, \mathrm{p}=0.034)$ and five years postoperatively (see figure 3 ) for the Catondeschamps ratio ( 0.77 vs $0.84, p=0.012$ ) (see figure 4 ) these may not be clinically significant given that these measures are dependent on where the tibia is resected.
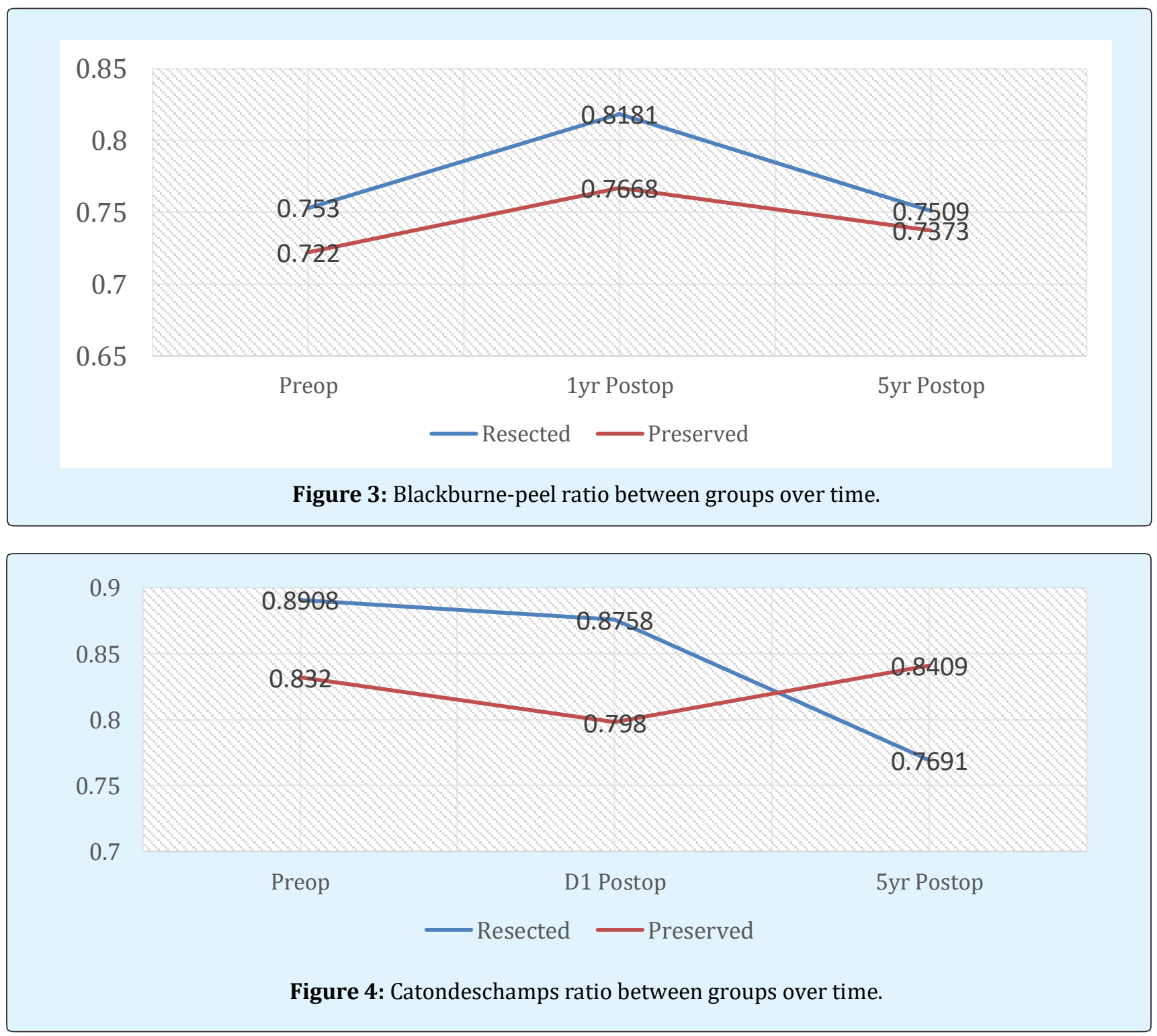

Knee society scores (KSS) preoperatively (45.36 vs $40.5, \mathrm{p}=0.31$ ) and at five years postoperatively ( 90.49 vs 85.31, $\mathrm{p}=0.2$ ) were not statistically different. Similarly anterior knee pain scores (AKPS) demonstrated no statistical significance between groups preoperatively ( 50.6 vs $42.8, \mathrm{p}=0.3$ ) and at five years post operatively (69.4 vs $62.0, \mathrm{p}=0.88$ ). 


\section{Journal of Orthopedics \& Bone Disorders}
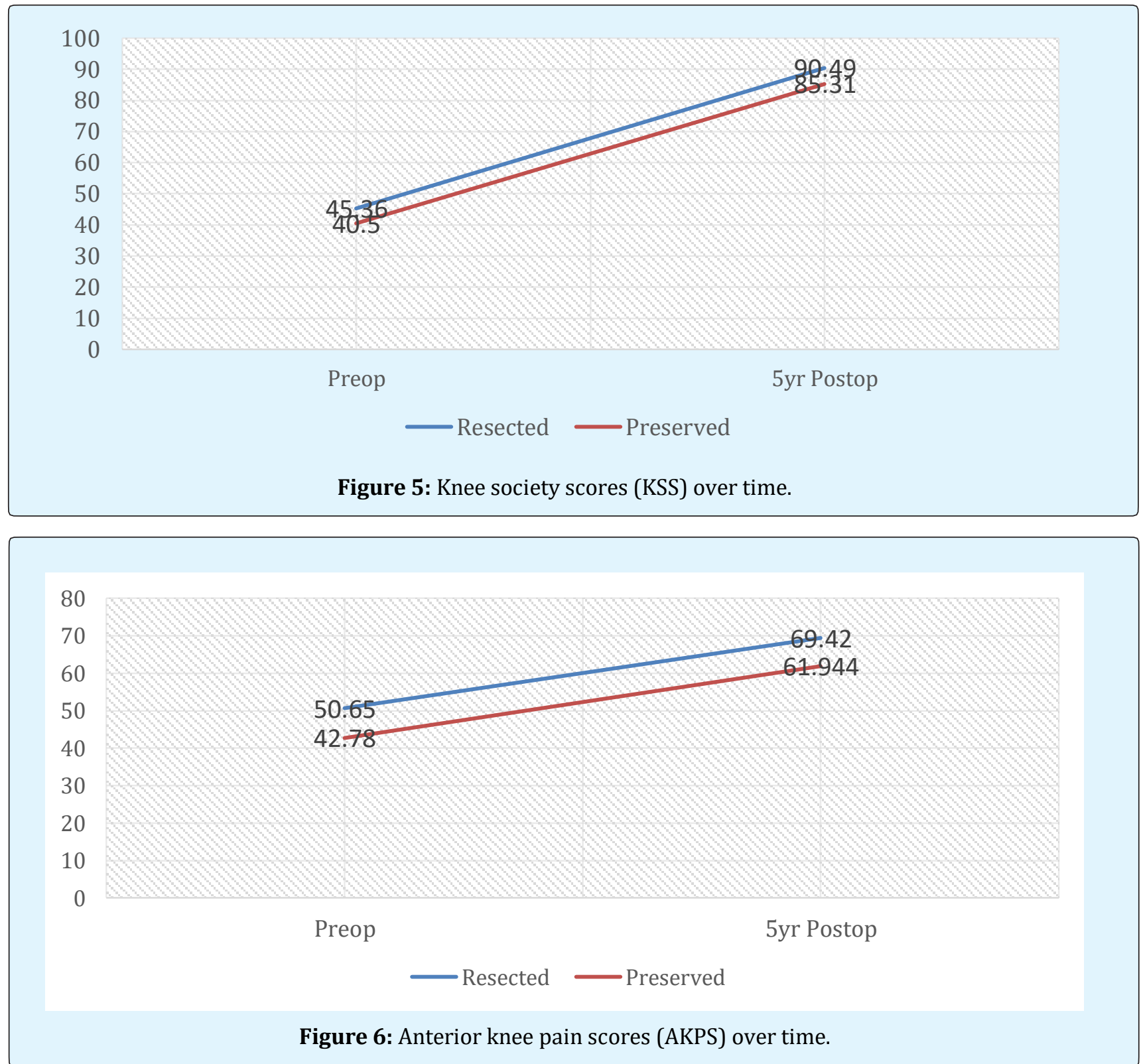

A binary logistical regression analysis was performed for Age $(>55,>65,>75)$, BMI $(<25,<30,<40)$ and Patella resurfacing (Yes, No). None of the above subsets predicted group membership.

\section{Discussion}

It remains unclear from the literature as to whether IPFP resection contributes to patella tendon shortening. Macule, et al. [14] conducted a randomized controlled trial assessing patellar tendon length at 5 days, 1 and 6 months postoperatively across two groups; one in which IPFP was excised and one in which it was preserved. Macule, et al. [14] found no significant difference in patellar tendon length between the two groups ( $p>0.05)$ [14]. Pinsornsak, et al. [15] made the same observation in their randomized controlled trial in patients up to 12 months follow-up [15].

Lemon et al had a longer follow-up period of 1-3years post TKR. They observed a statistically significant shortening of the patella tendon $(p=0.004)$ in the group 


\section{Journal of Orthopedics \& Bone Disorders}

that had the IPFP resected [16]. Tanaka, et al. [7] observed statistically significant shortening of the patella tendon at 28-38months post TKR in the IPFP resection group $(6.6 \mathrm{~mm}$ shortening, $\mathrm{p}<0.05)$, whilst the IPFP preservation group did not have significant tendon shortening (3.9mm, p>0.05) [7]. Gwyn, et al. [17] found complete excision of the fat pad had a significant shortening effect on the tendon in their retrospective radiological study [17]. However, Meneghini, et al. [18] had conflicting findings in their large retrospective study $(\mathrm{n}=1055)$. They found no significant difference in patellar tendon length at 5.1 years post-TKA whether the IPFP was preserved or resected [18].

The literature is divided on the effect of IPFP resection on anterior knee pain. Pinsornsak, et al. [15] found after a minimum of 6-months post-op there was a significantly higher incidence of anterior knee pain in TKRs where the IPFP was resected compared to when it was preserved [15]. Meneghini, et al. [18] made the same observation at 5.1 years follow-up [18]. Tanaka, et al. [7] also found a higher incidence of anterior knee pain in the resected group [7]. Conversely, Macule, et al. [14] observed higher rates of anterior knee pain amongst the preservation group at 6-months post op [14].

Similarly, there have been mixed findings on the effect of excising the IPFP on post-operative range of motion (ROM) in TKRs. At 1 year, Pinsornsak, et al. [15] found no difference in knee ROM between TKRs that had the IPFP resected and those in which the IPFP was preserved. Tanaka showed a statistically significant decreased ROM in the resection group at both 1-2 months and 28-38 months (both p <0.05) [7]. However, at 5.1-year follow-up, Meneghini, et al. observed no difference in ROM between the two groups [18].

This study demonstrates no significant difference in tendon length or functional outcome scores between resection and preservation of the infrapatellar fat pad in the setting of primary total knee replacement. The strengths of the study are that it was conducted at a single institution using a single implant in consecutive patients. Findings are somewhat limited given it is a retrospective study and does not take into account variations in patient comorbidities. Future prospective trials may be of benefit in confirming the results of this study and it may also be useful to compare other functional outcome scores such as the oxford knee score which has a component to address the effect of kneeling after arthroplasty surgery.

\section{Conclusion}

There is no statistical difference in radiological measures or functional scores (AKPS \&KSS) at one or five years post-operatively between groups having resection or preservation of the infrapatellar fat pad following total knee arthroplasty for primary osteoarthritis [22-25].

\section{References}

1. (2004) National Joint Registry for England and Wales: $1{ }^{\text {st }}$ Annual Report. National Joint Registry.

2. Scuderi GR, Windsor RE, Insall JN (1989) Observations on patellar height after proximal tibial osteotomy. J Bone Joint Surg Am 71(2): 245-248.

3. Dandy DJ, Desai SS (1994) Patellar tendon length after anterior cruciate ligament reconstruction. J Bone Joint Surg Br 76(2): 198.

4. O'Brien SJ, Warren RF, Pavlov H, Panariello R, Wickiewicz TL (1991) Reconstruction of the chronically insufficient anterior cruciate ligament with the central third of the patellar ligament. J Bone Joint Surg Am 73(2): 278-286.

5. Paulos LE, Rosenberg TD, Drawbert J, Manning J, Abbott P (1987) Infrapatellar contracture syndrome: an unrecognized cause of knee stiffness with patella entrapment and patella infera. Am J Sports Med 15(4): 331-341.

6. Weale AE, Murray DW, Newman JH, Ackroyd CE (1999) The length of the patellar tendon after unicompartmental and total knee replacement. J Bone Joint Surg Br 81(5): 790-795.

7. Koshino T, Ejima M, Okamoto R, Morii T (1990) Gradual low riding of the patella during postoperative course after total knee arthroplasty in osteoarthritis and rheumatoid arthritis. J Arthroplasty 5(4): 323327.

8. Tanaka N, Sakahashi H, Sato E, Hirose K, Isima T (2003) Influence of the infrapatellar fat pad resection in a synovectomy during total knee arthroplasty in patients with rheumatoid arthritis. J Arthroplasty 18(7): 897-902.

9. Figgie HE, Goldberg VM, Heiple KG, Moller HS, Gordon NH (1986) The influence of tibial-patellofemoral location on function of the knee in patients with the 


\section{Journal of Orthopedics \& Bone Disorders}

posterior stabilized condylar knee prosthesis. J Bone Joint Surg Am 68(7): 1035-1040.

10. Wojtys EM, Oakes B, Lindenfeld TN, Bach BR (1997) Patella infera syndrome: an analysis of the patellar tendon pathology. Instr Course Lect 46: 241-250.

11. Noyes FR, Wojtys EM, Marshall MT (1991) The early diagnosis and treatment of developmental patella infera syndrome. Clin Orthop 265: 241-252.

12. McMahon MS, Scuderi GR, Glashow JL, Scharf SC, Meltzer LP, et al. (1990) Scintigraphic determination of patellar viability after excision of infrapatellar fat pad and/or lateral retinacular release in total knee arthroplasty. Clin Orthop Relat Res 260: 10-6.

13. Kayler DE, Lyttle D (1988) Surgical interruption of patellar blood supply by total knee arthroplasty. Clin Orthop Relat Res 229: 221-227

14. Macule F, Sastre S, Lasurt S, Sala P, Segur JM, et al. (2005) Hoffa's fat pad resection in total knee arthroplasty. Acta Orthop Belg 71(6): 714-717.

15. Pinsornsak P, Naratrikun K, Chumchuen S (2014) The effect of infrapatellar fat pad excision on complications after minimally invasive TKA: a randomized controlled trial. Clin Orthop Relat Res 472(2): 695-701.

16. Lemon M, Packham I, Narang K, Craig DM (2007) Patellar tendon length after knee arthroplasty with and without preservation of the infrapatellar fat pad. J Arthroplasty 22(4): 574-580.

17. Gwyn R, Kotwal RS, Holt MD, Davies AP (2016) Complete excision of the infrapatellar fat pad is associated with patellar tendon shortening after primary total knee arthroplasty. Eur J Orthop Surg Traumatol 26(5): 545-549.
18. Meneghini RM, Pierson JL, Bagsby D, Berend ME, Ritter MA, et al. (2007) The effect of retropatellar fat pad excision on patellar tendon contracture and functional outcomes after total knee arthroplasty. J Arthroplasty 22(6 S2): 47-50.

19. White L, Holyoak R, Sant J, Hartnell N, Mullan J (2016) The effect of infrapatellar fat pad resection on outcomes post-total knee arthroplasty: a systematic review. Arch Orthop Trauma Surg 136(5): 701-708.

20. Van Beeck A, Clockaerts S, Somville J, Van Heeswijk JH, Van Glabbeek F, et al. (2013) Does infrapatellar fat pad resection in total knee arthroplasty impair clinical outcome? A systematic review. Knee 20(4): 226-231.

21. Baker PN, van der Meulen JH, Lewsey J, Gregg PJ, National Joint Registry for England and Wales (2007) The role of pain and function in determining patient satisfaction after total knee replacement. Data from the National Joint Registry for England and Wales. J Bone Joint Surg Br 89(7): 893-900.

22. Insall J, Salvati E (1971) Patella position in the normal knee joint. Radiology 101(1): 101-104

23. Blackburne JS, Peel TE (1977) A new method of measuring patellar height. J Bone Joint Surg 59(2): 241-242.

24. Caton J, Deschamps G, Chambat P, Lerat JL, Dejour H (1982) Les rotules basses A propos de 128 observations. Rev Chir Orthop 68(5): 317-325.

25. Noyes FR, Wojtys EM, Marshall MT (1991) The early diagnosis and treatment of developmental patella infera syndrome. Clin Orthop 265: 241-252. 